\title{
Collagen III (Reticulin) - A Location Marker of Pre-Sinusoid Arteriole Sphincters in the Villous System of Human Placenta
}

\author{
Gheorghe S Drăgoi ${ }^{1,2^{*}}$, Elena Pătrașcu ${ }^{2}$, Ileana Marinescu ${ }^{2}$, Elena Dincă ${ }^{2}$ and Petru Răzvan Melinte ${ }^{2}$ \\ ${ }^{1}$ Romanian Academy of Medical Sciences, Bucharest, Romania \\ 2 University of Medicine and Pharmacy of Craiova, Romania
}

*Corresponding author: Gheorghe S Drăgoi, Romanian Academy of Medical Sciences, University of Medicine and Pharrnacy of Craiova, Romania, Tel: 0040744547056; E-mail: dragoigs@gmail.com

Received date: February 22, 2017; Accepted date: March 20, 2017; Published date: March 24, 2017

Copyright: ( 2017 Dragoi GS, et al. This is an open-access article distributed under the terms of the Creative Commons Attribution License, which permits unrestricted use, distribution, and reproduction in any medium, provided the original author and source are credited.

\begin{abstract}
Blood micro-circulation within the placental villous system still raises a large number of problems of functional anatomy concerning the angio-structure of terminal villi, the communication between arterioles and venules of the umbilical vascular system, the spatial extension of the "extra-vascular contractile system" and, last but not least, the existence of the pre-sinusoid arteriole sphincters. The authors make a micro-anatomic analysis of the spatial distribution of the collagen fibres type III (reticulin) as markers of the pre-sinusoid arteriole sphincters. The study was performed on 20 human placentae of gestation age between 25 and 35 weeks. After paraffin embedding, 10 serial sections were obtained from each paraffin block. They were stained in Hematoxilin Eosin, or silver-impregnated according to Gömöri method. We had two objectives: to evaluate the spatial distribution of post arteriole sinusoid blood vessels within the terminal villi in the placenta, on the one hand, and the visualization of the location of a presinusoid arteriole sphincter system, on the other. The authors believe that umbilical arterioles and venules are connected "serially" at the level of terminal villi through arteriole-venule sinusoid blood vessels. The location of the trajectory of collagen type III (reticulin) bundles enveloping pre-sinusoid arterioles are markers of the existence of a pre-sinusoid arteriole sphincter at this level.
\end{abstract}

Keywords: Collagen type III (reticulin); Pre-sinusoid arteriole sphincter; Terminal villi; Mature intermediate villi; Sinusoid blood vessels

\section{Introduction}

The angioarchitecture of placental villi is a fascinating field of the functional anatomy of the foetus - placenta system. Although the foetus placenta blood circulation is anatomically autonomous from that of the mother, they are functionally interdependent. The hemodynamics in the placenta villous system still raises a large number of functional anatomy problems, i.e.

- What does the vascular structure of the human placenta terminal villi look like? Is there a capillary micro-circulation through a sinusoid, looping blood vessel that directly joins the arteriole and the venule of the umbilical vascular system?

- How is spatial communication between arterioles and venules at the level of the terminal villi realized: "serially" or in "parallel"? What are their functional influences on the blood flow?

- Can we view the blood circulation in the terminal villi as an "extraordinary arteriole - venule system"?

- Is there a spatial extension of the structures of the "extravascular contractile system" in the mature intermediate and/or the terminal villi?

- What are the relationships between the collagen III (reticulin) fibres and the contractile elements within the biodynamic structures of placenta villi?

In the course of the history of functional anatomy of the human placenta villouse system, the concept of "extravascular contractile structure" has evolved. Initially the cells similar to those in the smooth muscle were defined [1-4] and later these contractile elements were integrated in the "extra-vascular contractile system" at the level of stem villi, associated with the extra-cellular matrix of villose stroma $[5,6]$. The correlations between smooth muscle cells and the elastic fibres with the collagen fibres in a myofibroelastic anatomical-functional unit are well known [6].

The presence of pre-capillary sphincters was proved in many studies of micro-anatomy and physiology. Chamber and Zweifach [7], working on rabbit mesentery, identified the functional involvement of meta-arterioles in the hemodynamics in the capillary network. In his study on micro-circulation at the level of cat mesentery, Zweifach $[8,9]$ has confirmed, anatomically and functionally, the presence of precapillary sphincters.

The next question was whether pre-capillary sphincters and metaarterioles are universal components of tissular microcirculations $[10,11]$. A large number of physiology, pharmacology and microanatomy research have proved the existence of these structures in: striated somatic muscle [12-15]; integumentum commune [16,17] ; subcutaneous fat tissue [18]; gastric mucosa [19]; liver [20]; heart [21] and in the small intestine submucosa [22].

We started from the assumption that, at the level of terminal villi there is a communication system between the arterioles and umbilical venules through sinusoid blood vessels. To control the blood flow within the terminal villi the presence of a pre-sinusoid arteriole sphincter system regulating the flow is required.

In the present paper, the authors aim at visualizing the pre-sinusoid arteriole sphincter location by impregnating with silver the collagen type III fibre (reticulin) bundles. The object of the paper concerns the 
spatial distribution of the sinusoid blood vessels within the terminal villosities on the one hand, and the distribution of the collagen fibres type III as markers of the location of the pre-sinusoid arteriole sphincters, on the other.

\section{Materials and Method}

The study was conducted on human biologic material, respecting the norms of the Code of Medical Ethics and the Code for Scientific Research Ethics. The micro-anatomic study of the placental structures was performed on 20 human placentae (gestation age 25 to 35 weeks) processed shortly after deliverance inside Hospital Filantropia of Craiova, Departments Obstetric-Gynecologie and Pathology. They were washed in physiological saline solution $9 \%$ and immersed in buffered formaldehyde $5 \%, \mathrm{pH}$ 7.4. Three days after embedding tissular fragments of $2 / 2 \mathrm{~cm}$ are taken from the para-central and marginal zones of the placenta discus. After re-embedding in histological paraffin, 10 serial sections (for $5 \mu \mathrm{m}$ ) were cut from each paraffin block. The sections were either colored in Hematoxilin- Eosin for general histo-topographic orientation in order to evaluate the angioarchitecture of placenta villosities, or silver-impregnated according to Gömöri method in order to visualize the collagen fibres type III (reticulin) bundles. Micro-photography was performed on microscope Nikon 80 i by means of Sight DS-Fil Hight Definition Color Camera Head and processed in Software NIS - Elements Advanced Research

\section{Results}

Based on the microanatomic analysis of the serial sections of placenta, the authors had two objectives in view:

- Evaluation of the spatial distribution of post-arteriole sinusoid blood vessels in the terminal villi;

- Visualization of the location through an argirophilic collagen marker, at the level of the arterioles existing in mature intermediate villi, of a pre-sinusoid arteriole sphincter system.

\section{Micro-anatomic analysis of the spatial distribution of arteriole sinusoids in terminal villosities}

The examination in X10 objective and X40 objective of the placenta terminal villi connected to the mature intermediate villi enabled the authors to evaluate their form and angiostructure. The form is determined by the intravillouse, vascular and stromal anatomic structures on the one hand, and their biunivocal interconnections. The location of intravillouse blood vessels is variable: marginal in cortex villi, central in the centrum villi (Figures $1 \mathrm{C}, 1 \mathrm{D}, 1 \mathrm{~F}$ and $1 \mathrm{G}$ ).

The external surface of the terminal villi, lined with syncytiotrophoblasts is bosselated by prominances reflecting the presence of marginal sinusoid blood vessels. The form of terminal villi is similar to that of a bunch of grapes (Figures $1 \mathrm{~A}-1 \mathrm{D}, 1 \mathrm{~F}$ and $1 \mathrm{G}$ ). Marginal blood vessels compress the syncytiotrophoblast and participate in the formation of the syncytial - vascular membrane (Figure $1 \mathrm{H}$ ). It is difficult to establish - in micro-anatomic sections the appurtnance of intravillouse sinusoid blood vessels to the advehent/in-coming arteriole revehent/out-going venous systems.

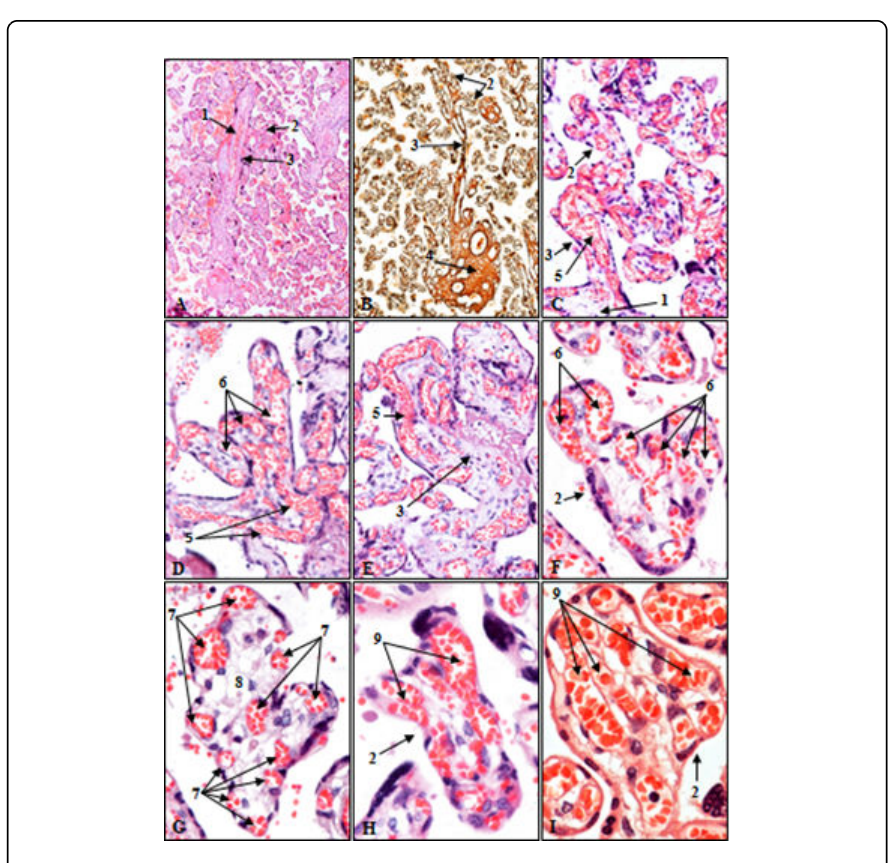

Figure 1: Spatial distribution of pre-sinusoid arterioles into mature intermediate villi and of sinusoid blood vessels into terminal villi of human placenta. 1: Stem villi; 2: Terminal villi; 3: Mature intermediate villi; 4: Villous tree; 5: Pre-sinusoid arteriole within the mature intermediate villi, longitudinal section; 6: Sinusoid blood vessel, within the terminal villi, oblique section; 7: Sinusoid blood vessel within terminal villi, transverse section; 8: Villous stroma; 9: Loop - trajectory blood vessel within the terminal villi, longitudinal section; 10: Syncytial vascular membrane. Paraffin sections, Hematoxylin Eosin stain (A and C - I); Reduced Silver Nitrat Gömöri Method (B). Magnifier: Large image X10 (A,B), X140 (CE); X420 (F-I).

\section{Micro - anatomic analysis of the location and trajectory of collagen fibres type III (reticulin) bundles at the level of pre- sinusoid arterioles in intermediary mature villosities}

The examination in X10 objective and X40 objective made us easily identify pre-sinusoid arterioles from their origins in the trunkular arteries of stem villi to the terminal villi. Crossing the mature intermediate villi, pre-sinusoid penetrate the terminal villi where they are continuous with the arteriole sinusoids, and through the latter with the in-coming sinusoids of the post-sinusoid venules, without possible micro-anatomic personalization (Figures 2A-2D). The sinusoid trajectory of pre-sinusoid arterioles and of arteriole and venule sinusoid vessels is clearer on the serial sections in varied planes of the terminal villi and/or mature intermediate villi: frontal, saggital, paramedial and/or horizontal (Figures 2A,2B,2E,2G and 2H).

The examination in X20, X40, X60 obiectives of silver-impregnated serial sections - Gömöri method enabled the visualization of the location and trajectory of the bundles of collagen fibres type III (reticulin) at the level of pre-sinusoid arterioles and of the villous stroma (Figures 2B,2D-2F and $2 \mathrm{H}$ ). At the level of pre-sinusoid arterioles we saw dark-colored, argerophilic strips/bands alternating with clear, nonsilver-impregnated ones. This image suggests that at this 
level there is a pre-sinusoid arteriole sphincter (Figures $2 \mathrm{~B}, 2 \mathrm{D}, 2 \mathrm{~F}$ and $2 \mathrm{H})$.

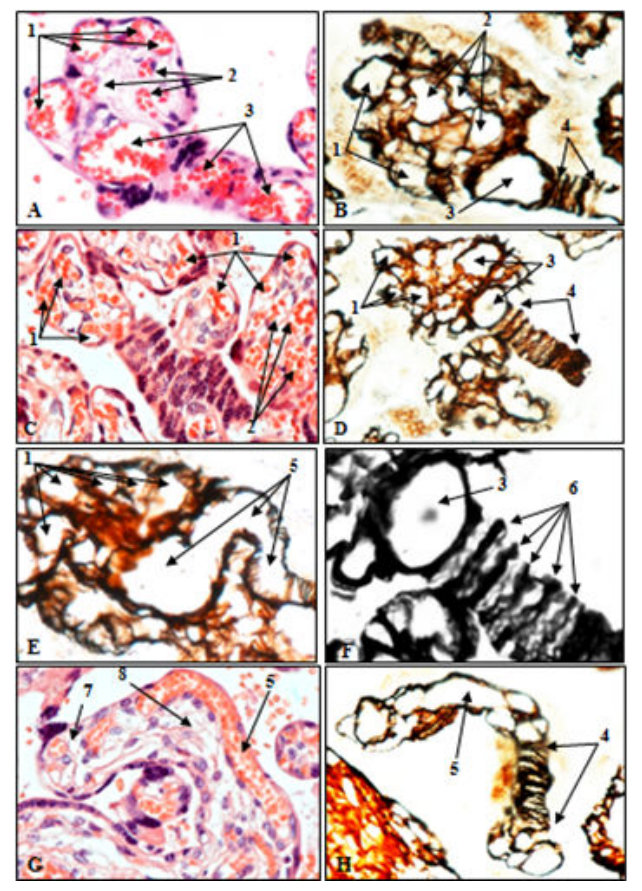

Figure 2: Location and trajectory of bundles of collagen fibres type III (reticulin), at the level of pre-sinusoid arterioles, in mature intermediate villi. 1: Sinusoid blood vessels in the terminal villi cortex; 2: Sinusoid blood vessels in the center of terminal villi; 3 : Pre-sinusoid arteriole, in transversal section; 4: Pre-sinusoid arteriolar sphincter; 5: Pre-sinusoid arteriole, in longitudinal section; 6: Bundles of collagen fibres type III (reticulin) of looping trajectory, at the level of pre-sinusoid arteriole; 7: Terminal villi; 8: Stroma in the central part of mature intermediate villi. Paraffin section. Hematoxilin Eosin stain $(A, C, G)$. Reduced Silver Nitrat Gömöri Method (B,D,E,F,H). Magnifier: X140 (A,D), X280 (B,C,H), X420 (E-G).

\section{Discussion}

Based on the previous information, we could attempt a few answers to some of the issues that blood biodynamics in the placenta villous system is confronted with.

\section{Stereo distribution of sinusoid blood vessels in villous micro- circulation}

Blood micro-circulation in the placental villous system takes place in the absence of a true capillary network between arterioles and venules in the umbilical vessels. It is achieved through sinusoid blood vessels in the terminal villi. However, Bernischke si Kaufmannn [23] use two terms for the intravillous blood vessels: capillaries and sinusoids. They define sinusoids as dilated capillaries of approximately $50 \mu \mathrm{m}$. We suggest that umbilical arterioles and venules are "serially" connected through an anastomosis arteriole-venule by means of arteriole-venule sinusoids. The absence of an interposed capillary network between arterioles and venules is replaced by post-arteriole sinusoid blood vessels distributed fanlike. Thus, we can talk about an "extraordinary arteriole-venule sinusoid system". This anatomic characteristic allows the growth of the transversal section of the intravillous vascular bed and, implicitly, a decrease of resistance to the blood flow, according to Laplace law: the flow speed is reverse to the transversal section in the blood vessel. This is the mechanism through which the conditions for the mother-Foetus nutrients and gas exchanges are made possible at the level of the syncytial-vascular membrane.

\section{Pre-sinusoid sphincters in the mature intermediate villi system}

Pre-capillary sphincters described by Rhodin (1967) [24] in varied tissues as complete perivascular sleeves have not been identified in human placenta [24]. It is thought that arterioles are continuous with 1-2 capillaries by the gradual decrese of arteriole diameter which are free from smooth muscle cells [25].

The authors have visualized bundles of collagen fibres type III (reticulin) having circumferential and spiral trajectory at the level of the pre-sinusoid arteriole in the mature intermediate villi, this image suggesting the existence of a pre-sinusoid arteriole sphincter at this level. Graf et al. (1994) [5] have described "perivascular contractile sheaths" in the stem villi, also called "extra-vascular contractile system". Ultrastructural studies have suggested the association at this level of smooth muscle cells and subpopulations of microfibroblasts differentited from the perivascular mesenchimal stroma [26,27].

The presence and trajectory of collagen fibres type III (reticulin) we have identified at the level of mature intermediate villi, around the presinusoid arteriole, are markers of the existence of a pre-sinusoid sphincter at this level. It can be integrated in the "extravascular contractile system" as an extension of the latter from the stem villi into the mature intermediate villi.

\section{Allostasic regulation of placental blood flow}

"The remarkable sinusoid arteriole" venular system located in villi terminalis ensures the direct transition of the blood from umbilical arteries to the similar vene. Between these huge vascular streams there are sinusoidal capillary loops that should not be assimilated to a true capillary network.

The presence of pre-sinusoid arterial sphincters is a lead to a allostasic, holistic concept of placental blood flow stability by selfregulation $[28,29]$.

We believe that the allostatic regulation of placental blood flow is achieved by changes, multifactorially determined, of the villose functional structures: variations of pre-sinusoid arteriole diameter, and, implicitely, of the sinusoids within the villi terminalis; phenotypical transformations of the villose stroma; villose intra and extra fibrin deposits; the existence of angio and vasculo-genetic processes, and, last but not least, of pathological states diabetes, hypoxia, pre-eclampsia, maternal malnutrition that involve the modulation of vascular resistance and entail restrictions in the foetal intrauterine growth. 


\section{Conclusion}

Pre-sinusoidal arteriolar sphincters are fibro-muscular structures involved in the allostatic self-regulation of placental blood flow stability;

The fascicles of collagen fibres type III (reticulin) visualized at the level of pre-sinusoidal arterioles, by location and their spiral trajectory can be viewed as micro-anatomic markers of the existence of presinusoidal sphincters;

Allostatic regulation of villose blood flow achieved through phenotypical transformations of villose structures as well as through the presence of pre-sinusoidal arteriolar sphincters involves modulation of vascular resistance with consequences upon the intrauterine growth of the foetus.

\section{References}

1. Iisuda S (1916) Concentration of muscle fibres in the human placenta. Beitr Geburtsh Gynaek 19: 101.

2. Naujoks H (1922) Do anatomical changes of the childish have an ejaculation influence on the time of the bubble jump? Z Geburtsh Gynaek 84: 304.

3. Dubreuil G, Rivière M (1932) Fibromuscular formations of the chorion and villus of the human placenta. CR Soc Biol 111: 170-172.

4. Spanner (1935) Maternal and cognitive circulation of the human placenta and its strombahnen. Z Anat Development 105: 163-242.

5. Graf R, Langer JU, Schöenfelder G, Oeney T, Hartel Schenk S, et al. (1994) The extravascular contractile system in the human placenta Morphological and immunohistochemical investigations. Anat Embryol 190: 541-548.

6. Graf R, Metejevic D, Schuppan D, Nuedeck H, Shakibaei M, et al. (1997) Molecular anatomy of the perivascular sheath in human placental stem villi : contractil apparatus and its association to the extracellular matrix. Cell Tissue Res 290: 601-607.

7. Chambers R, Zweifach BW (1994) Topography and function of the mesenteric capillary circulation. Am J Anat 75: 173-205.

8. Zweifach BW (1949) Basic mechanisn in peripheral homeostasis. In : Zweifach BW (eds) Factors regulating blood pressure. Transactions of the third conference, New York, Josiac Macy, Jr Fundation pp: 13-52.

9. Zweifach BW, Shorr E, Black MM (1953) The influence of the adrenal cortex on behavior of terminal vascular bed. Ann NY Acad Sci 56 626-633.

10. Johnson LR (2003) Essential medical physiology. Elsevier Academic Press, MA, USA.

11. Boron WF, Boulpaep EL (2008) Medical Physiology. (2 ${ }^{\text {nd }}$ edn), Philadedlphia, Elsevier. Sauders, Philadelphia, USA.

12. Mellander S (1966) Comparative effects of acetylcholine, butyl-nordynephrine (Vascular), noradrenaline and ethyl-adrainol (Effonti) on resistance, capacitance and precapillary sphincter vessels and capillary filtatration in cat skeletal muscle. Angiologica 3: 77-99.

13. Owen DA, Stuerner E (1971) Effect of dihydroergotamine (DHG) on the capacitance, resistance and precapillary sphincter vessels of denervated cat skeletal muscle. Br J Pharmacol. 42: 655-656.

14. Hillman J, Lundvall J (1981) Classification of beta-adrenoceptors in the microcirculation of sckeletal muscle. Acta Physiol Scand 113: 67-71.

15. Bentzer P. Kongstad L, Grande PO (2001) Capillary filtration coefficient in independent of number of perfused capillaries in cat skeletal muscle. Am J Physiol 280: 2697-2706.

16. Tooke JE (1980) A capillary pressure disturbance in young diabetics. Diabetes 29: 815-819.

17. Widmer RJ, Laurinec JE, Joung MF, Mohiddin MW, Laine GA, et al. (2008) The origin of the biphasic flow response to local heat in skin. Microcirculation 15: 349- 357.

18. Burcher E, Olgart L, Gazelius B (1977) Comparative effects of adrenaline and felypressin (octapressin) on consecutive sections of the vascular bed in canine adipose. Acta Physiol Scand 100: 215-220.

19. Perry MA, Granger DN (1985) Regulation of capillary exchange capacity in the dog stomach. Am J Physiol 248: 437-442.

20. Oda M, Han JY, Yokomori H (2000) Local regulators of hepatic sinusoid microcirculation : recent advances. Clin Hemorrheol Microcirc 23: 85-94.

21. Schneeweiss A, Sherf L, Lehrer E, Lieberman Y, Neufeld HN (1982) Segmental study of the terminal coronary vessels in coarctation of the aorta : a natural model of the effect of coronary hypertension on human coronary circulation. Am J Cardiol 49: 1996-2002.

22. Granger DN, Kvietys PR, Perry MA, Barrowman JA (1987) The microcirculation and intestinal transport. In: Johnson LR (eds) Physiology of the Gastrointestinal Tract. ( $2^{\text {nd }}$ edn), Raven Press, New York, USA pp: 1671-1697.

23. Rhodin JAG (1967) The ultrastructure of mammalian arterioles and precapillary sphinters. Ultrastruct $18: 181-223$.

24. Benirschke K, Kaufmann P (2000) Pathology of the human placenta, $\left(4^{\text {th }}\right.$ Edn) Springer-Verlag New York, USA pp: 116-148.

25. Kaufmann P, Bruns U, Leiser R Luckhandt M, Winterhager E (1985) The Foetal vascularisation of term placental villi. II. Intermediate and terminal villi. Anat Embryol 173: 203-214.

26. Kohen G, Kertschanska S, Demir R, Kaufmann P (1996) Placental villous stroma as a model system for myofibroblast differentiation. Histochem Cell Biol 105: 415-429.

27. Demir R, Kosanke G, Kertschanska S, Kaufmann P (1997) Classification of human placental stem villi: review of structural and functional aspects. Microsc Res Tech 38: 29-41.

28. McEwen BS, Wingfield JC (2003) The concept of allostasis in biology and biomedicine. Horm Behav 43: 2-15.

29. Sterling P, Allostasis EJ (1988) A new paradigm to explain arousal pathology. In: Fisher S, Reason J (eds) Handbook of life stress, Cognition and health. John Wiley\&Sons, New York, USA pp: 629-649. 